\title{
Computer assisted research: Using SYM for the symmetry analysis of Differential Equations
}

\author{
Stylianos Dimas \\ CMCC - Centro de Matemática, Computação e Cognição, UFABC, \\ 09210 - 170, Santo André, SP \\ E-mail: s.dimas@ufabc.edu.br.
}

\begin{abstract}
$\underline{\text { Abstract }}$
SYM is a symbolic package - developed in the computer algebra system Mathematica - for the symmetry analysis of Differential Equations (DE) [5, 8]. And since its initial concept on 2002 it is in constant development by the author.

The purpose of the package is to function as a symbolic toolbox of commands for the symmetry analysis, and manipulation of those symmetries, in the study of DE. It consists of fully automated commands and low level ones in order to cover a wide range of tasks: from automatically retrieve the classical Lie point symmetries of a DE to interactively obtain the approximate symmetries or constructing conservation laws using the previously found symmetries of a DE to name a few. Since its first presentation back at 2005, [3], many new commands were added augmenting its functionality. Currently, SYM consists of more than a hundred commands divided loosely into there categories: Symmetry analysis, Lie Algebra analysis and Auxiliary commands.

In the present poster an exposition of the advancements of the package since 2005 will be given. Apart from a general review of its structure and its basic functionality the following aspects of the package will be illustrated:

- Analysis of the algebraic properties of the symmetries of a DE, solvability, nullity, derived series, radical, quotient space, change of variables Levi decomposition etc..

- Equivalence transformations: group classification of classes of DE containing arbitrary parameters and functions $[8,6,9]$. We show how to obtain the simplest representative of a class of DE simplifying hence its classification.

- Approximate symmetries: symmetry analysis of perturbed DEs from the point of view of the perturbation theory $[1,4,2]$. We consider three different ways and obtain perturbed closed solutions for each one of them.

- Conservation laws: construction of conservation laws using the symmetries of a DE, even when the DE does not have a variational form or is of even order [7]. Classification of a class of DE by the self-adjointness property and non-trivial conservation laws.
\end{abstract}

Keywords: computer assisted research, symmetries of DE, conservation laws

\section{References}

[1] V. A. Baikov, R. K. Gazizov and N. H. Ibragimov, Approximate symmetries, Matematicheskii Sbornik, 178 (1988) 435-450. 
[2] G. I. Burde, On the Use of the Lie Group Technique for Differential Equations with a Small Parameter: Approximate Solutions and Integrable Equations, Physics of Atomic Nuclei, 65 (2002) 990-995.

[3] S. Dimas and D. Tsoubelis, SYM: A new symmetry-finding package for Mathematica, In "The $10^{\text {th }}$ International Conference in MOdern GRoup ANalysis" (N. Ibragimov, C. Sophocleous and P. Damianou, eds.) pp. 64-70, University of Cyprus, Nicosia, 2005.

[4] W. I. Fushchich and W. M. Shtelen, On approximate symmetry and approximate solutions of the nonlinear wave equation with a small parameter, J. Phys. A: Math. Gen., 22 (1989) L887.

[5] N. H. Ibragimov, "Transformation Groups Applied to Mathematical Physics", Mathematics and its Applications Springer, ${ }^{\text {st }}$ ed., 1985.

[6] N. H. Ibragimov, Equivalence groups and invariants of linear and nonlinear equations, Archives of ALGA, 4 (2009) 41-100.

[7] N. H. Ibragimov, Nonlinear self-adjointness in constructing conservation laws, In "Archives of ALGA" (N. H. Ibragimov, ed.), vol. 7/8 ALGA publications, Karlskrona, Sweden (2010-2011) pp. $1-90$.

[8] L. Ovsiannikov, "Group Analysis of Differential Equations", Academic Press, $1^{\text {st }}$ ed., 1982, 432 pages.

[9] R. O. Popovych and H. Eshraghi, Admissible Point Transformations of Nonlinear Schrödinger Equations, In "Proceedings of the $10^{\text {th }}$ International Conference in MOdern GRoup ANalysis" (N. Ibragimov, C. Sophocleous and P. Damianou, eds.) (2005) pp. 167-174. 